\title{
Toxicidade do dimetilsulfóxido em cães hígidos e doentes renais crônicos
}

\author{
Toxicity of dimethyl sulfoxide in healthy dogs and with chronic kidney disease
}

\section{Leandro Zuccolotto Crivellenti ${ }^{{ }^{*}}$ Sofia Borin Crivellenti ${ }^{\mathrm{I}}$ Marileda Bonafim Carvalho ${ }^{\mathrm{II}}$}

RESUMO

O objetivo do presente trabalho foi avaliar os efeitos do tratamento com dimetilsulfóxido (DMSO) sobre aspectos pertinentes à função renal, perfil bioquímico sérico, parâmetros hematológicos e condição clínica de cães sadios e de cães com doença renal crônica (DRC). As avaliações foram feitas antes, durante e após a administração de DMSO a $10 \%$ na dose de $0,5 \mathrm{~g} \mathrm{~kg}^{-1}$, cada $24 \mathrm{~h}$, por três dias. O DMSO resultou em alguns efeitos adversos nos cães sadios e também nos cães com DRC, nos quais os efeitos foram mais frequentes e mais graves. Apesar desses efeitos adversos, tanto em cães sadios quanto para cães com DRC 2-3, não há contraindicações para o uso do fármaco quando em curto espaço de tempo. A gravidade dos efeitos adversos relacionados ao tratamento com DMSO e possível associação com o óbito em cães com DRC em estádio 4, constituem fatores para contraindicação do fármaco nesse grupo de pacientes.

Palavras-chave: taxa de filtração glomerular, depuração de creatinina, excreção fracionada de eletrólitos, função renal.

\section{ABSTRACT}

The objective of this study was to evaluate the effects of dimethyl sulfoxide (DMSO) treatment on aspects of renal function, serum profile, total blood count parameters and clinical condition of health or chronic kidney disease (CKD) dogs. The evaluations were done before, during and after the administration of DMSO $10 \%$ at a dose of $0.5 \mathrm{~g} \mathrm{~kg}^{-1}$, each $24 \mathrm{~h}$, for three days. DMSO resulted in some adverse effects in both healthy and CKD dogs, however the effects were more frequent and worse in CKD dogs. Despite these adverse effects, both groups don't have contraindications to use the drug in a short time. The severity of adverse effects related to the DMSO and its possible association with death in stage 4 CKD dogs, are contraindications for the drug in this group of patients.

Key words: glomerular filtration rate, creatinine clearance, electrolytes fractional excretion, renal function.

\section{INTRODUÇÃO}

O dimetilsulfóxido (DMSO) é um solvente amplamente utilizado para diluição de medicamentos hidro e lipossolúveis e como preservante celular, além de ser empregado como medicamento para redução da pressão intracraniana e de lesões do sistema nervoso central, dentre outras aplicações (MASTRO et al., 2001; JACOB \& TORRE, 2009). As propriedades farmacológicas do DMSO incluem ação carreadora e potencializadora de outros fármacos, anti-inflamatória, vasodilatadora (STONE, 1993), antioxidante, analgésica, diurética, imunomoduladora, miorrelaxante, antimicrobiana e citoprotetora (MASTRO et al., 2001; JO et al., 2004; JACOB \& TORRE, 2009). Apesar das controvérsias, há várias décadas, o DMSO vem sendo empregado na medicina e medicina veterinária. Independentemente da via de administração, a maior parte do produto é excretada por via urinária como dimetilsulfóxido inalterado e, em menor quantidade, sob a forma de dimetilsulfona, além do dimetilsulfato que é excretado por via respiratória. Quando indicado e sob dosagem correta, o DMSO aparentemente não resulta em efeitos adversos relevantes (BRAYTON, 1986; ORLATO, 2006).

Contudo, assim como ocorre com diversos fármacos, tal condição pode ser modificada em pacientes com doença renal crônica (DRC) sempre que houver comprometimento da função renal excretora e alterações extrarrenais decorrentes da

IPrograma de pos-graduação em Medicina Veterinária, Faculdade de Ciências Agrárias e Veterinárias (FCAV), Universidade Estadual

Paulista (UNESP), 14884-900, Jaboticabal, SP, Brasil. E-mail: crivellenti_lz@yahoo.com.br.*Autor para correspondência.

IDDepartamento de Clínica e Cirurgia Veterinária (FCAV/UNESP), Jaboticabal, SP, Brasil. 
insuficiência renal e uremia. Nesses casos, podem ocorrer alterações na distribuição, biotransformação e excreção do fármaco ou de seus metabólitos e, consequentemente, o aparecimento de reações adversas, incluindo nefrotoxicidade.

Embora existam relatos esporádicos relacionados à toxicidade do DMSO em pacientes, tanto na medicina quanto na medicina veterinária, até a atualidade, o tema não foi objeto de experimento controlado. Assim, buscou-se avaliar parâmetros fisiológicos e variáveis laboratoriais pertinentes às funções renal, hepática e hematológica de animais sadios e portadores de DRC, submetidos ao tratamento com DMSO na menor dose recomendada (BRAYTON, 1986).

\section{MATERIAL E MÉTODOS}

O protocolo experimental para o presente estudo foi aprovado pela Comissão de Ética no Uso de Animais da FCAV/UNESP (processo n.00613609) em 26 de abril de 2009. Utilizaram-se cães com DRC e cães sadios provenientes do canil experimental e do canil experimental e do Serviço de Nefrologia e Urologia do Hospital Veterinário UNESP - campus de Jaboticabal. Os animais foram mantidos em gaiolas metabólicas, desde 48 horas antes do início das avaliações (adaptação), com água ad libitum e ração oferecida duas vezes ao dia e lá permaneceram até o término da avaliação pósimediata. Entre as avaliações pós-imediata e tardia, os animais mantiveram-se em domicílio, sem o uso de medicamentos ou qualquer outra substância e, na avaliação tardia, foram seguidos os mesmos procedimentos anteriores.

Os cães foram submetidos ao tratamento com DMSO a 10\% (DMSO diluído em solução de cloreto de sódio a $0,9 \%$ ), na dose de $0,5 \mathrm{~g} \mathrm{~kg}^{-1}$ por dia (realizado no período da manhã), por aplicação intravascular na velocidade de $10 \mathrm{~mL} \mathrm{~kg}^{-1} \mathrm{~h}^{-1}$, durante três dias consecutivos. Ao encerramento de cada ciclo de 24 horas, foram realizadas as coletas de dados e de amostras. Para investigar os efeitos do tratamento, foram avaliados débito urinário de 24 horas, clearance (depuração) de creatinina endógena de 24 horas (Ccr), pela fórmula $\mathrm{Ccr}\left(\mathrm{mL} \mathrm{min} \mathrm{mg}^{-1}\right)=\left\{\left[\mathrm{Ucr}\left(\mathrm{mg} \mathrm{mL} \mathrm{mL}^{-1}\right)\right.\right.$ x Uv(mL) $\left.] \div\left[\operatorname{Scr}\left(\mathrm{mg} \mathrm{dL}^{-1}\right) \mathrm{x} \mathrm{T}(\min )\right]\right\} \div \operatorname{peso}(\mathrm{kg})$, excreção urinária de proteínas (razão proteína/ creatinina urinária e proteína de 24 horas) (método sensiprot e Jaffé modificado, respectivamente), excreção fracionada de sódio, em urina de 24 horas (EFNa) (método de íons-seletivos) e as concentrações séricas de creatinina (método Jaffé modificado), ureia (método enzimático), cálcio (reação cromogênica com cresolftaleina complexona), fósforo (método de Daly e Ertingshausen modificado), sódio e potássio (método de íons-seletivos), proteína total (método biureto), albumina (método verde de bromocresol), alanina-aminotransferase (método Reitman e Frankel), fosfatase alcalina (método Roy modificado), colesterol (método Enzimático de Trinder), além de eritrograma e pressão arterial sistólica (método doppler).

Ao longo do período de estudo, houve acompanhamento clínico e exame físico diário dos cães. A primeira avaliação - basal - foi feita no dia anterior ao do início do tratamento com DMSO (prétratamento); seguiram-se avaliações nos dias 1, 2 e 3 (tratamento), nos dias 4 e 5 (pós-tratamento imediato) e no dia 30 (pós-tratamento tardio) (Figura 1).

De acordo com a condição clínicolaboratorial inicial, os animais foram classificados em três grupos. Seis cães sadios compuseram o grupo controle e outros 10 cães nefropatas crônicos compuseram os grupos de doentes, sendo um grupo de seis cães com DRC em estádios 2 e 3 (DRC 2-3) e outro de quatro cães com DRC em estádio 4 (DRC 4). A classificação do estádio da DRC foi feita de acordo com o preconizado pela International Renal Interest Society (IRIS - Staging CKD, 2006).

O trabalho seguiu um delineamento em blocos casualizados, tendo como critério de exclusão, para o grupo DRC, a existência de infecção do trato urinário, crise urêmica e qualquer comorbidade. Os resultados foram submetidos à análise de variância (ANOVA) tendo como fatores de variação o grupo (controle e DRC 2-3) e o tempo (sete avaliações). Para comparação de médias, foi empregado o teste de comparações múltiplas de Tukey-Kramer. Valores de $\mathrm{P} \leq 0,05$ foram considerados significativos. Os cálculos foram feitos por meio do programa estatístico $S A S^{\circledR}$ (Statistical Analysis System). Os dados dos animais do grupo DRC 4 não foram incluídos na análise estatística, mas foram considerados individualmente.

\section{RESULTADOS E DISCUSSÃO}

A condição clínico-laboratorial inicial dos cães com DRC 2-3, tendo como referência os dados do grupo de cães sadios, foi caracterizada por diminuição do Ccr, aumento da produção de urina e da proteinúria, além de azotemia. Alguns paciente apresentaram EFNa $>1 \%$, hipertensão, anemia e hiperfosfatemia (Tabela 1).

Diferentemente dos trabalhos que relataram a possibilidade de resultados promissores 


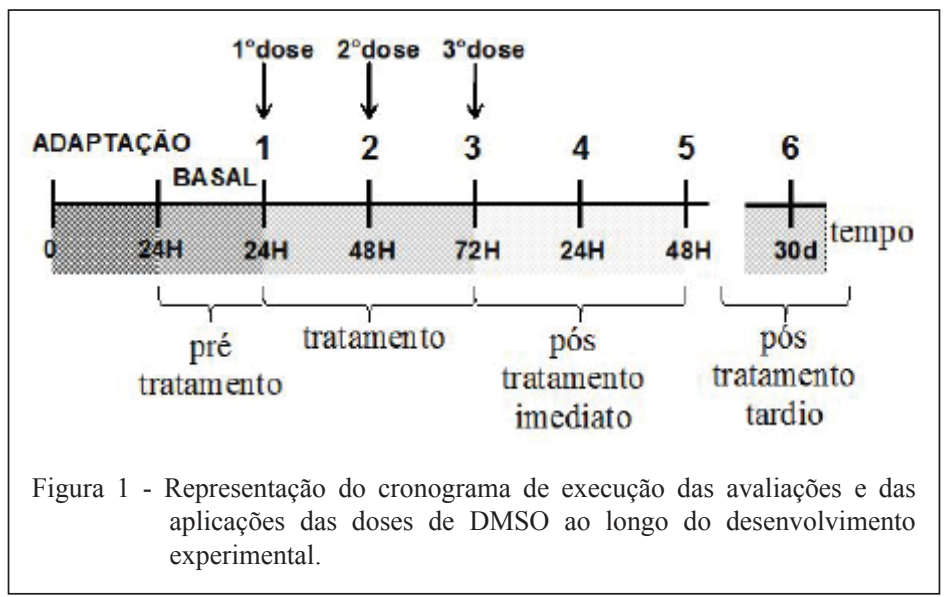

na sobrevida e na diminuição da azotemia com uso do DMSO no tratamento de lesões renais, sejam elas decorrentes de isquemia (KEDAR et al., 1983), nefrotoxinas (ALI \& MOUSA, 2001; JO et al., 2004) ou glomerulopatias induzidas (LOTAN et al., 1984), os pacientes com DRC desse experimento não apresentaram redução significativa das concentrações séricas de creatinina e ureia. Entretanto, no presente experimento, os cães examinados eram doentes renais crônicos já urêmicos.

Quando avaliado em coelhos neonatos, o DMSO foi capaz de aumentar a TFG até certo ponto, pois doses elevadas causaram redução significativa do fluxo sanguíneo renal, aumento da resistência vascular renal e consequente diminuição da produção de urina (RIJITEMA et al., 1999). Segundo ORLATO (2006), a administração de DMSO na dose de $1 \mathrm{~g}$ $\mathrm{kg}^{-1}$ a cada 12 horas durante três dias foi capaz de aumentar o clearance de creatinina de cães sadios. Embora isso não possa ser dito sobre os cães sadios do experimento em tela, observou-se tendência ao aumento dessa variável nos animais com DRC 2-3 (Figura 2A).

A U-P/C apresenta alta correlação com a proteinúria de 24 horas, considerada a melhor metodologia para avaliação da perda protéica renal (LEES, 2004). No presente estudo, avaliou-se a proteinúria de 24 horas, por ser mais fidedigna, e a U-P/C, por sua praticidade na aplicação clínica. Embora os cães DRC tenham apresentado proteinúria maior que os sadios, não houve alteração significativa ao longo do estudo. Achado semelhante foi obtido por ORLATO (2006), em estudo realizado com cães sadios. A hipercolesterolemia pode estar associada com os quadros de proteinúria acentuada em pacientes com DRC marcada por lesão glomerular (POLZIN et al., 1997). De forma semelhante à da proteinúria, o colesterol sérico parece não ter sofrido influência do tratamento com DMSO, apesar de apresentar-se em maior concentração nos DRC, quando comparado ao controle, nos período de tratamento e pós-tratamento imediato

Dentre os indicadores da função tubular, a excreção fracionada de sódio é a mais conhecida e pode ser utilizada clinicamente para diferenciar azotemias pré-renais das renais, casos de nefrites tubulointersticiais e doenças renais crônicas, especialmente quando a taxa de filtração glomerular já se encontra diminuída (BURANAKARL et al., 2007). Durante o processo de evolução da DRC, a fim de manter o balanço eletrolítico, os túbulos diminuem a reabsorção de sódio, aumentando gradativamente sua concentração na urina (ZATZ, 2002). Neste trabalho, observou-se que a excreção fracionada de sódio foi influenciada pelo fator doença, pois se apresentou maior no grupo DRC, principalmente nos do estádio 4. Supõe-se que a tendência ao aumento da excreção fracionada de sódio e do volume urinário durante o período de tratamento com o DMSO (figura 2B), embora não significativa, seja advinda das propriedades diuréticas do fármaco (BRAYTON, 1986; STONE, 1993).

As concentrações séricas de fósforo (basal) foram maiores nos cães com DRC, com destaque para os pacientes em estádio 4 (Tabela 1), e foram observadas diferenças significativas entre o grupo DRC 2-3 e o grupo controle nos períodos de tratamento e póstratamento imediato (Tabela 2), embora esses achados não tenham sido correlacionados ao aumento de creatinina, supõem-se que possam ter ocorrido tanto pelos efeitos adversos (vômito, diminuição de apetite, diarreia, etc.) quanto por ação direta do fármaco, causando a diminuição da excreção do fósforo. A hiperfosfatemia pode promover o aparecimento do hiperparatireoidismo, mineralização de tecidos e progressão da DRC, eventos diretamente relacionados

Ciência Rural, v.43, n.10, out, 2013. 
Tabela 1 - Médias e erro padrão das primeiras avaliações (basal) que caracterizaram as condições clínico-laboratoriais dos cães estudados dados do grupo controle, do grupo DRC em estádio 2 ou 3 (DRC 2-3), e do grupo DRC em estádio 4 (DRC 4).

\begin{tabular}{|c|c|c|c|c|c|c|}
\hline \multirow{3}{*}{ Parâmetros } & \multirow{3}{*}{ Controle } & \multirow{3}{*}{ DRC 2-3 } & \multicolumn{4}{|c|}{ 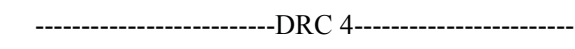 } \\
\hline & & & \multicolumn{4}{|c|}{ 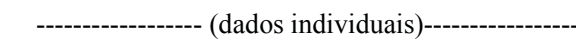 } \\
\hline & & & 1 & 2 & 3 & 4 \\
\hline \multicolumn{7}{|l|}{ Função renal } \\
\hline Volume urinário $\left(\mathrm{mL} \mathrm{kg}^{-1} 24 \mathrm{~h}^{-1}\right)$ & $13,7 \pm 2,0^{\mathrm{a}}$ & $46,4 \pm 7,4^{b}$ & 44,4 & 62,3 & 60,4 & 54,2 \\
\hline Clearance de creatinina $\left(\mathrm{mL} \min ^{-1} \mathrm{~kg}^{-1}\right)$ & $2,1 \pm 0,3^{\mathrm{a}}$ & $1,2 \pm 0,2^{\mathrm{b}}$ & 0,3 & 0,4 & 0,3 & 0,3 \\
\hline Razão proteína/creatinina urinária & $0,2 \pm 0,1^{\mathrm{a}}$ & $1,2 \pm 0,3^{\mathrm{b}}$ & 2,1 & 1,6 & 3,0 & 3,3 \\
\hline Proteína urinária $\left(\mathrm{mg} \mathrm{kg}^{-1} 24 \mathrm{~h}^{-1}\right)$ & $4,7 \pm 1,8^{\mathrm{a}}$ & $55,2 \pm 17,8^{b}$ & 47,5 & 88,7 & 94,3 & 99,8 \\
\hline Excreção fracionada de sódio (\%) & $0,3 \pm 0,1^{\mathrm{a}}$ & $1,3 \pm 0,4^{\mathrm{b}}$ & 5,1 & 3,9 & 10,1 & 9,1 \\
\hline \multicolumn{7}{|l|}{ Perfil sérico } \\
\hline Creatinina $\left(\mathrm{mg} \mathrm{dL}^{-1}\right)$ & $1,1 \pm 0,1^{\mathrm{a}}$ & $2,4 \pm 0,4^{\mathrm{b}}$ & 5,4 & 8,3 & 7,2 & 7,9 \\
\hline Ureia $\left(\mathrm{mg} \mathrm{dL}^{-1}\right)$ & $30,0 \pm 4,8^{\mathrm{a}}$ & $109,1 \pm 17,3^{\mathrm{b}}$ & 239,4 & 209,2 & 371,0 & 341,6 \\
\hline Cálcio total (mg dL $\left.{ }^{-1}\right)$ & $9,7 \pm 0,6^{\mathrm{a}}$ & $10,8 \pm 0,5^{\mathrm{a}}$ & 10,7 & 11,3 & - & 10,8 \\
\hline Fósforo $\left(\mathrm{mg} \mathrm{dL}^{-1}\right)$ & $4,5 \pm 0,5^{\mathrm{a}}$ & $6,1 \pm 1,0^{\mathrm{a}}$ & 6,7 & 14,4 & 19,5 & 16,5 \\
\hline Sódio $\left(\mathrm{mmol} \mathrm{L}^{-1}\right)$ & $140,5 \pm 2,7^{\mathrm{a}}$ & $139,3 \pm 0,7^{\mathrm{a}}$ & 142 & 135 & 147 & 142 \\
\hline Potássio $\left(\mathrm{mmol} \mathrm{L}^{-1}\right)$ & $4,7 \pm 0,2^{\mathrm{a}}$ & $4,4 \pm 0,1^{\mathrm{a}}$ & 4,8 & 4,6 & 5,0 & 4,1 \\
\hline Proteína Total $\left(\mathrm{mg} \mathrm{dL}^{-1}\right)$ & $7,4 \pm 0,3^{\mathrm{a}}$ & $8,0 \pm 1,2^{\mathrm{a}}$ & 6,7 & 6,7 & 5,7 & 5,8 \\
\hline Albumina $\left(\mathrm{mg} \mathrm{dL}^{-1}\right)$ & $3,4 \pm 0,2^{\mathrm{a}}$ & $2,1 \pm 0,1^{\mathrm{b}}$ & 2,6 & 3,3 & 1,3 & 2,0 \\
\hline Alanina aminotransferase $\left(\mathrm{U} \mathrm{L}^{-1}\right)$ & $80,9 \pm 15,7^{\mathrm{a}}$ & $64,0 \pm 15,9^{\mathrm{a}}$ & 26,2 & 138,7 & 136,2 & 47,1 \\
\hline Fosfatase alcalina $\left(\mathrm{U} \mathrm{L}^{-1}\right)$ & $103,1 \pm 24,0^{\mathrm{a}}$ & $102,4 \pm 30,5^{\mathrm{a}}$ & 37,6 & 103,3 & 75,1 & 28,2 \\
\hline Colesterol $\left(\mathrm{mg} \mathrm{dL}^{-1}\right)$ & $187,0 \pm 21,5^{\mathrm{a}}$ & $228,5 \pm 26,1^{\mathrm{a}}$ & 321 & 272 & 168 & 198 \\
\hline \multicolumn{7}{|l|}{ Hemograma } \\
\hline Hemácias $\left(\times 10^{6} \mu \mathrm{L}^{-1}\right)$ & $6,7 \pm 0,3^{\mathrm{a}}$ & $5,3 \pm 0,5^{\mathrm{b}}$ & 6,3 & 4,1 & 2,9 & 3,0 \\
\hline Hemoglobina $\left(\mathrm{g} \mathrm{dL}^{-1}\right)$ & $15,6 \pm 0,6^{\mathrm{a}}$ & $11,5 \pm 1,1^{\mathrm{b}}$ & 12,7 & 9,2 & 6,2 & 7,2 \\
\hline Hematócrito $(\%)$ & $47,6 \pm 1,7^{\mathrm{a}}$ & $38,4 \pm 3,2^{\mathrm{b}}$ & 39,5 & 28,2 & 19,4 & 21,2 \\
\hline Plaquetas $\left(\times 10^{3} \mu \mathrm{L}^{-1}\right)$ & $308,3 \pm 22,0^{\mathrm{a}}$ & $385,8 \pm 63,7^{\mathrm{a}}$ & 118 & 501 & 312 & 122 \\
\hline Leucócitos $\left(\times 10^{3} \mu \mathrm{L}^{-1}\right)$ & $7,2 \pm 0,6^{\mathrm{a}}$ & $10,5 \pm 1,6^{\mathrm{a}}$ & 11,5 & 11,0 & 16,1 & 9,8 \\
\hline Pressão arterial sistólica (mmHg) & $142,5 \pm 10,1^{\mathrm{a}}$ & $165,0 \pm 12,8^{\mathrm{a}}$ & 180 & 220 & 260 & 240 \\
\hline
\end{tabular}

* Médias seguidas de diferentes letras na mesma linha diferenciam-se estatisticamente entre si.

com aumento da mortalidade em humanos, cães e gatos (POLZIN, 2011). Dentre os cães DRC 4, os três casos de óbito estiveram associados com hiperfosfatemia mais grave, corroborando os dados da literatura.

A anemia, um dos achados mais comuns em cães com DRC (POLZIN et al., 1997; CRIVELLENTI, 2012), pode ser agravada por lesões do trato gastrintestinal com perda de sangue (POLZIN, 2011) e também pela fragilidade dos eritrócitos e redução de sua vida útil, consequentes à modificação da proporção de fosfolípides e de colesterol na membrana eritrocitária (AKMAL et al., 1985), além dos outros fatores relacionados à eritropoiese. De fato, neste trabalho, foram observados números menores de hemácias, hemoglobina e hematócrito no grupo DRC, quando comparado ao grupo controle. De acordo com Stone (1993), somente o DMSO em concentrações superiores a $20 \%$ pode causar hemólise, porém observou-se diferença significativa entre $\mathrm{o}$ pós-tratamento imediato $(\mathrm{He}=4,96 \pm 0,53$ e $\mathrm{Ht}=36,58 \pm 2,86)$ e pós-tratamento tardio $(\mathrm{He}=5,39 \pm 0,60$ e $\mathrm{Ht}=40,00 \pm 4,14)$ nos animais com DRC 2 e 3 em relação aos parâmetros hemácias e hematócrito, respectivamente. Embora essa ocorrência possa estar associada à hemólise durante o período de tratamento, provavelmente secundária à fragilidade eritrocitária supracitada, a hipótese de que se trate de consequência da perda sanguínea no trato digestório, em decorrência da hematoquesia e ao fato de que não houve alterações significativas no VCM e CHCM, parece ser mais plausível.

Os sinais clínicos e achados laboratoriais dos pacientes com DRC podem ter variação ampla, dependendo da velocidade de progressão, natureza e duração do processo patológico (POLZIN et al., 1997; LEES, 2004). Mas também é possível que, independente do estádio da DRC, o paciente encontre-se clinicamente estável, como era o caso dos que foram estudados. Embora os sinais clínicos 


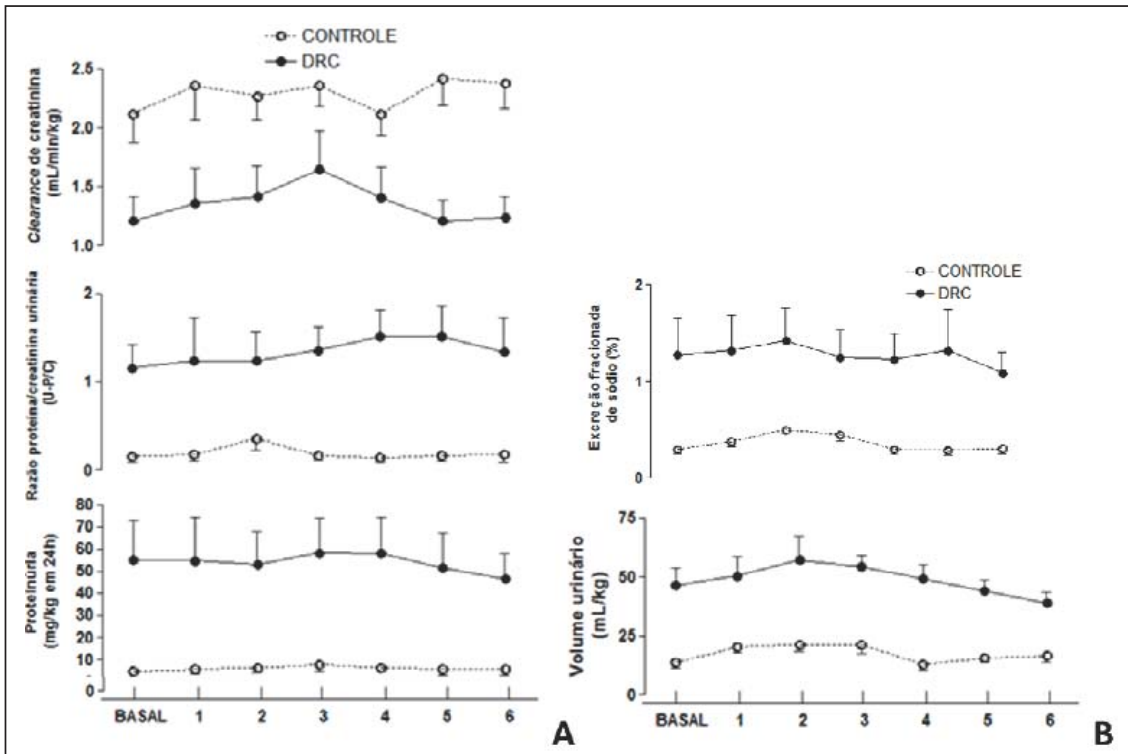

Figura 2 - Representação gráfica (A) clearance de creatinina, U-P/C e proteinúria de 24 horas e (B) excreção fracionada de sódio e volume urinário do grupo controle $(\mathrm{n}=6)$ e grupo DRC 2-3 $(n=6)$ ao longo do cronograma experimental. Barras acima e abaixo dos pontos indicadores de média representam o erro padrão.

manifestados a partir do início do tratamento tenham sido os mesmos descritos na síndrome urêmica (apatia, diarreia, hematoquesia e vômito) (POLZIN et al., 1997; CRIVELLENTI, 2012), possivelmente foram desencadeados pelo DMSO.

Inúmeros efeitos adversos já foram associados à administração de doses letais e semi-letais do DMSO, dentre os quais se destacam diminuição na atividade motora espontânea, tremores, dispneia, estupor, hipotermia, convulsões e morte (SMITH et al., 1967). Devido às semelhanças dos sinais clínicos apresentados pelos doentes renais crônicos após a administração do DMSO e da intensificação destes nos DRC 4, sugere-se que, quanto mais avançado o estádio da doença renal, maiores são as chances de ocorrerem efeitos adversos ao fármaco. Três dos quatro animais do grupo DRC 4, tratados com DMSO, neste experimento, apresentaram tremores, dispneia, diarreia, hematoquesia, vômito, hipotermia e morte.

POLZIN et al. (1997) descreveram que a hiporexia manifestada pelos DRC pode ser exacerbada por alterações ambientais, as quais frequentemente resultam em redução da ingestão hídrica, desidratação e piora do quadro. No experimento em questão, observou-se que o apetite dos animais do grupo DRC 2-3 não foi influenciado pelo DMSO, porém alguns animais do grupo controle e DRC 4 apresentaram hiporexia ou anorexia, em alguns momentos do tratamento. Redução do apetite já havia sido relatada por LOTAN et al. (1984) em ratos tratados com DMSO, contudo, devido à instabilidade clínica dos cães DRC 4, a ocorrência de anorexia neste grupo não pode ser totalmente atribuída aos efeitos do DMSO.

A hipertensão arterial encontra-se entre as complicações mais comuns da DRC, chegando a ocorrer em 50 a 93\% dos casos (POLZIN et al., 1997). Corroborando os dados da literatura, verificou-se que a maioria $(70 \%)$ dos animais com doença renal crônica deste trabalho era hipertenso, com destaque para os DRC 4 (100\%). Mas não foram detectadas variações relacionadas ao tratamento com DMSO.

\section{CONCLUSÃO}

O DMSO administrado na dose de $0,5 \mathrm{~g} \mathrm{~kg}^{-1}$, IV, a cada 24 horas, por três dias consecutivos, pode resultar em alguns efeitos adversos leves em cães sadios e também em cães com DRC e, nesses, a intensidade, tipo e frequência de ocorrência são maiores.

Considerando que o DMSO pode ser indicado para diversas modalidades terapêuticas, os resultados do presente estudo permitem concluir que, no que se refere à função renal, tanto para cães sadios quanto para cães com DRC 2-3, não há contraindicações para o uso do fármaco quando em curto espaço de tempo. A gravidade dos efeitos adversos relacionados ao tratamento com DMSO e possível associação com o óbito em cães com DRC 4, constatadas neste estudo, constituem fatores de contraindicação para uso do fármaco. 
Tabela 2 - Média \pm erro padrão do perfil bioquímico sérico das concentrações de cálcio total e fósforo. Resultados obtidos em sete avaliações do grupo controle $(n=6)$ e grupo DRC 2-3 $(n=6)$, tratados com DMSO

\begin{tabular}{|c|c|c|c|c|}
\hline \multirow{2}{*}{ Variáveis } & \multirow{2}{*}{ Períodos } & \multirow{2}{*}{ Avaliações } & \multicolumn{2}{|c|}{--------------------Grupos-------------------- } \\
\hline & & & Controle & DRC 2-3 \\
\hline \multirow{7}{*}{$\begin{array}{l}\text { Cálcio total } \\
\left(\mathrm{mg} \mathrm{dL}^{-1}\right)\end{array}$} & \multirow[t]{2}{*}{ Pré-tratamento } & BASAL & $9,65 \pm 0,56$ & $10,77 \pm 0,45$ \\
\hline & & 1 & $11,05 \pm 0,36$ & $10,25 \pm 0,41$ \\
\hline & \multirow[t]{2}{*}{ Tratamento } & 2 & $11,12 \pm 0,72$ & $10,17 \pm 0,51$ \\
\hline & & 3 & $10,32 \pm 0,30$ & $10,35 \pm 0,46$ \\
\hline & \multirow{2}{*}{ Pós-tratamento imediato } & 4 & $9,97 \pm 0,29$ & $10,25 \pm 0,58$ \\
\hline & & 5 & $10,03 \pm 0,30$ & $10,22 \pm 0,39$ \\
\hline & Pós-tratamento tardio & 6 & $9,80 \pm 0,35$ & $10,28 \pm 0,43$ \\
\hline \multirow{7}{*}{$\begin{array}{l}\text { Fósforo } \\
\left(\mathrm{mg} \mathrm{dL}^{-1}\right)\end{array}$} & Pré-tratamento & BASAL & $4,53 \pm 0,45$ & $6,07 \pm 1,04$ \\
\hline & \multirow{3}{*}{ Tratamento } & 1 & $4,75 \pm 0,46^{\mathrm{a}}$ & $6,22 \pm 1,02^{\mathrm{b}}$ \\
\hline & & 2 & $4,40 \pm 0,21^{\mathrm{a}}$ & $6,12 \pm 1,00^{\mathrm{b}}$ \\
\hline & & 3 & $4,42 \pm 0,21^{\mathrm{a}}$ & $7,32 \pm 1,79^{\mathrm{b}}$ \\
\hline & \multirow{2}{*}{ Pós-tratamento imediato } & 4 & $4,51 \pm 0,25^{\mathrm{a}}$ & $6,58 \pm 0,83^{\mathrm{b}}$ \\
\hline & & 5 & $4,74 \pm 0,35^{\mathrm{a}}$ & $6,32 \pm 1,05^{\mathrm{b}}$ \\
\hline & Pós-tratamento tardio & 6 & $4,62 \pm 0,28$ & $5,60 \pm 0,90$ \\
\hline
\end{tabular}

* Médias seguidas de diferentes letras na mesma linha diferenciam-se estatisticamente entre si.

\section{AGRADECIMENTOS}

À Fundação de Amparo à Pesquisa do Estado de São Paulo (FAPESP) e ao Conselho Nacional de Pesquisa e Tecnologia (CNPq), pela Bolsa de Mestrado e pelo Custeio do Projeto, respectivamente.

\section{REFERÊNCIAS}

AKMAL, M. et al. Erythrocyte survival in chronic renal failure role of secondary hyperparathyroidism. Journal of Clinical Investigation, New Haven, v.76, p.1695-1698, 1985. Disponível em: <http://www.ncbi.nlm.nih.gov/pmc/articles/PMC424168/pdf/ jcinvest00124-0413.pdf>. Acesso em: jul. 2011.

ALI, B. H.; MOUSA, H.M. Effect of dimethyl sulfoxide on gentamicin induced nephrotoxicity in rats. Human \& Experimental Toxicology, London, v.20, p.199-203, 2001. Disponível em: $<$ http://het.sagepub.com/content/20/4/199.abstract $>$. Acesso em: dez. 2011. doi: 10.1191/096032701678766859.

BRAYTON, C.F. Dimethyl sulfoxide (DMSO): a review. Cornell Veterinarian, Ithaca, v.76, n.1, p.76-90, 1986

BURANAKARL, C. et al. Relationships between degree of azotaemia and blood pressure, urinary protein:creatinine ratio and fractional excretion of electrolytes in dogs with renal azotaemia. Veterinary Research Communications, Amsterdam, v.31, p.245257, 2007. Disponível em: <http://www.springerlink.com/content/ x773403912h32724/fulltext.pdf>. Acesso em: fev. 2011. doi: 10.1007/s11259-006-3413-2.

CRIVELLENTI, L.Z. Nefrologia e urologia. In: CRIVELLENTI, L.Z.; BORIN-CRIVELLENTI, S. Casos de rotina em medicina veterinária de pequenos animais. São Paulo: MedVet, 2012. Cap.9, p.255-304.

JACOB, S.W.; TORRE, J.C. Pharmacology of dimethyl sulfoxide in cardiac and CNS damage. Pharmacological Reports, Kraków, v.61, p.225-235, 2009. Disponível em: <http://rabbit.if-pan.krakow. pl/pjp/pdf/2009/2_225.pdf $>$. Acesso em: dez. 2011.

JO, S.K. et al. Delayed DMSO administration protects the kidney from mercuric chloride-induced injury. Journal of the American Society of Nephrology, Baltimore, v.15, p.2648-2654, 2004. Disponível em: $<$ http://jasn.asnjournals.org/content/15/10/2648.full.pdf + html $>$. Acesso em: 20 dez. 2011. doi: 10.1097/01.ASN.0000139933.20109.CB.

KEDAR, I. et al. Dimethyl sulfoxide in acute ischemia of the kidney. Annals of the New York Academy of Sciences, New York. v.411, p.131-134, 1983. Acesso em: dez. 2011. doi: 10.1111/j.17496632.1983.tb47294.x

LEES, G.E. Early diagnosis of renal disease and renal failure. Veterinary Clinics of North America: Small Animal Practice, Philadelphia, v.34, p.867, 2004

LOTAN, D. et al. Reduction of protein excretion by dimethyl sulfoxide in rats with passive heymann nephritis. Kidney International, New York, v.25, p.778-788, 1984. Disponível em: <http://www.nature. com/ki/journal/v25/n5/pdf/ki198490a.pdf>. Acesso em: dez. 2011. doi: 10.1038/ki.1984.90.

MASTRO, L.D. et al. Intensified chemotherapy supported by DMSOfree peripheral blood progenitor cells in breast cancer patients. Annals of Oncology, London, v.12, p.505-508, 2001. Disponível em: <http:// annonc.oxfordjournals.org/content/12/4/505.full.pdf $+h t m l>$. Acesso em: fev. 2012.

ORLATO, D. Efeitos do DMSO (dimetilsulfóxido), administrado por via intravenosa, sobre as funções renal e hepática, perfil hidrossalino e hemograma de cães sadios. 2006. 50f. Dissertação (Mestrado em Cirurgia Veterinária) - Faculdade Ciências Agrárias e Veterinárias, Universidade Estadual Paulista "Julio de Mesquita Filho", Jaboticabal, SP.

POLZIN, D.J. Chronic kidney disease in small animals. Veterinary Clinics of North America: Small Animal Practice, Philadelphia, v.41, p.15-30, 2011.

Ciência Rural, v.43, n.10, out, 2013. 
POLZIN, D.J. et al. Insuficiência renal crônica. In: ETTINGER, S.J.; FELDMAN, E.C. Tratado de medicina interna. 4.ed. São Paulo: Manole, 1997. V.2, cap.134, p.2394-2431.

RIJITEMA, M. et al. The effects of dimethyl sulfoxide on renal function of the newborn rabbit. Biology of the Neonate, Basel, v.76, p.355-361, 1999. Disponível em: <http://content.karger.com/ ProdukteDB/produkte.asp?Aktion=ShowPDF\&ArtikelNr $=1417$ 9\&ProduktNr=225294\&filename=14179.pdf $>$. Acesso em: dez 2011. doi: 10.1159/000014179.
SMITH, E.R. et al. The single and repeated dose toxicity of dimethyl sulfoxide. Annals of the New York Academy of Sciences, New York, v.141, p.96-109, 1967. Acesso em: dez. 2011. doi: 10.1111/j.1749-6632.1967.tb34870.x.

STONE, R.W. Clinical updates on the use of dimethyl sulfoxide. Canine Practice, Santa Barbara. v.18, p.16-18, 1993.

ZATZ, R. Distúrbios da filtração glomerular. In: Fisiopatologia renal. 2.ed. São Paulo: Atheneu, 2002. p.3-49. 